\title{
MiR-139 suppresses $\beta$-casein synthesis and proliferation in bovine mammary epithelial cells by targeting the GHR and IGF1R signaling pathways
}

Yingjun Cui ${ }^{1,2}$, Xia Sun ${ }^{1}$, Lianfeng Jin ${ }^{1}$, Guangpu Yu ${ }^{1}$, Qingzhang $\mathrm{Li}^{1}$, Xuejun Gao ${ }^{1}$, Jinxia Ao ${ }^{2}$ and Chunmei Wang ${ }^{1 *}$

\begin{abstract}
Background: MicroRNAs have important roles in many biological processes. However, the role of miR-139 in healthy mammary gland remains unclear. The objective of this study was to investigate the effects of miR-139 on lactation in dairy cows.

Results: Here, we found that miR-139 was down-regulated in mid-lactation dairy cow mammary tissues compared with mid-pregnancy tissues. Then, we prioritized two of potential target genes of miR-139 in cow, growth hormone receptor (GHR) and type I insulin-like growth factor receptor (IGFIR) for further functional studies based on their roles in lactation processes. Dual luciferase reporter assays validated direct binding of miR-139 to the 3'untranslated region (UTR) of GHR and IGFIR. Moreover, over-expression or silencing of miR-139 affected mRNA levels of GHR and IGF1R in cultured bovine mammary epithelial cells (BMECs). Furthermore, over-expression of miR139 decreased protein levels of $\beta$-casein, proliferation in mammary epithelial cell, and the protein levels of IGF1R and key members of the GHR or IGF1R pathways as well, whereas silencing miR-139 produced the opposite result. Among these signal molecules, signal transducer and activator of transcription-5 (STAT5), protein kinase B (also known as AKT1), mammalian target of rapamycin (mTOR), and p70-S6 Kinase (p70S6K) are involed in $\beta$-casein synthesis, and Cyclin D1 is involved in cell proliferation. In addition, silencing GHR decreased protein levels of $\beta$ casein, IGF1R, and key members of the IGF1R pathway, whereas co-silencing miR-139 and GHR rescued the expression of GHR and reversed GHR silencing effects.
\end{abstract}

Conclusions: Our results demonstrate that GHR and IGFIR are target genes of miR-139 in dairy cow. MiR-139 suppresses $\beta$-casein synthesis and proliferation in BMECs by targeting the GHR and IGF1R signaling pathways.

Keywords: miR-139, Mammary gland, $\beta$-casein, Growth hormone receptor, Type I insulin-like growth factor receptor

\section{Background}

MicroRNA-139-5p (miR-139-5p) and miR-139-3p are mature miRNAs generated from a miR-139 precursor $[1,2]$. The knowledge about miR-139-3p function in bovine mammary epithelium is very limited, but it is worth noting that miR-139-3p has been identified in bovine milk [3]. It is a widely used assumption that there is only one type of mature bta-miR-139, and it

\footnotetext{
* Correspondence: wangcm-1@163.com

'Key Laboratory of Dairy Science of Education Ministry, Northeast Agricultural University, Harbin, Heilongjiang 150030, People's Republic of China

Full list of author information is available at the end of the article
}

has the same sequence as hsa-miR-139-5p. MicroRNAs regulate a wide variety of biological processes, including cell proliferation, apoptosis, developmental timing, and signal transduction [4]. Previous studies about miR-139 have focused on its antioncogenic and antimetastatic activities [5]. It has been shown that miR-139-5p suppresses proliferation, migration, and invasion in esophageal cancer [6], colorectal cancer [7], non-small cell lung cancer [8], hepatocellular carcinoma [9] and breast cancer [5, 10]. Moreover, miR139-5p inhibits the epithelial-mesenchymal transition of hepatocellular carcinoma cells [2]. Although some 
microarray studies show that miR-139 expression is down-regulated in the mammary tissue during the transition period between pregnancy and lactation [11], little is known about effects of miR-139 in healthy mammary glands.

Growth hormone $(\mathrm{GH})$ is a pituitary hormone that exerts a galactopoietic effect on the bovine mammary gland and controls milk protein synthesis [12, 13]. In addition to the pituitary gland, $\mathrm{GH}$ is produced in an autocrine manner by the mammary gland [14]. The effects of $\mathrm{GH}$ are mediated through interaction with growth hormone receptor (GHR), whose expression is regulated by GH $[14,15]$. Localization of GHR is strong on the membrane as well as in the cytoplasm of the bovine mammary epithelial cell (BMEC) $[15,16]$. GHR immunostaining within the nucleus is rare or absent [17]. GHR may be involved in milk protein secretion in BMECs [12]. The most abundant milk protein is $\beta$ casein, which makes up approximately $30 \%$ of the total protein in cows' milk [18].

Type I insulin-like growth factor (IGF1) is the major mediator of GH effects in many tissues. Previous studies have indicated that GH can act indirectly on the mammary gland by stimulating IGF1 secretion from the liver [19]. Evidence has also shown that GH induces the expression of IGF1 mRNA in mammary gland [20, 21]. The functions of IGF1 are mainly mediated through the type I insulin-like growth factor receptor (IGF1R) [22]. Interestingly, some of the IGF1R downstream signaling members are shared with GH [20]. IGF1R signaling can promote cell proliferation, survival, differentiation, and protein synthesis $[19,20]$.

In this study, GHR and IGF1R were prioritized as two of potential target genes of miR-139 in cow. Combining their roles in lactation processes, and the downregulation of miR-139 in the mammary tissue during the transition period between pregnancy and lactation, we hypothesized that miR-139 is also involved in regulation of lactation. The aim of this study was to investigate the effects of miR-139 on lactation in dairy cows.

\section{Methods}

\section{Animals and mammary gland samples}

Samples were collected from mammary glands of Holstein cows. All animals were clinically healthy. The average weights of the dairy cows were $609 \pm 9.08 \mathrm{~kg}$ (mean \pm SEM). The cows were separated into two groups by developmental stage: mid-pregnancy $(n=3)$ or midlactation $(n=3)$. Three pregnant cows went through a dry period and then were pregnant again. Lactating cows were at 90 DIM. The cows were slaughtered by exsanguination, and the mammary glands were removed immediately after slaughtering. Mammary gland samples were frozen in liquid nitrogen and stored at $-80{ }^{\circ} \mathrm{C}$. In our study, all animal experimental protocols were approved by the Institutional Animal Care and Use Committee of Northeast Agricultural University (China).

\section{Plasmid construction}

The wild-type and mutated sequences of the 3'- untranslated region (UTR) of GHR (Cow GHR, NCBI Accession \# NC_007318.5) and IGF1R (Cow IGF1R, NCBI Accession \# AC_000178.1) with specific restriction sites XhoI and NotI were constructed by Sangon Biotech Company (Shanghai, China).

The 214-bp fragment from GHR 3'-UTR contained a predicted bta-miR-139 binding region ( $\mathbf{5}^{\prime}$-ACACGGTG TACTGTAG-3'). The binding site of the mutated $3^{\prime}$ UTR sequence of GHR was 5'-TGTGCGTGTACA CATC-3'. The 242-bp fragment from IGF1R 3'-UTR contained a predicted bta-miR-139 binding region (5' ACTGTAGA-3'). The binding site of the mutated $3^{\prime}$ UTR sequence of IGF1R was 5' 'TGACATCT-3'. All sequences were confirmed by sequence analysis. The wildtype or mutant 3'-UTR was subcloned into the XhoINotI site of the psiCHECK-2 vector (Promega, Madison, WI, USA).

\section{HeLa cell culture and dual luciferase reporter assay}

The HeLa cell line was purchased from the American Type Culture Collection (ATCC, Rockville, MD, USA). The cell line was maintained in Dulbecco's modified Eagle's medium (DMEM) (High Glucose) (Invitrogen, Carlsbad, CA, USA) and supplemented with $10 \%$ fetal bovine serum (FBS) (Gibco, Rockville, Maryland, USA), penicillin (100 units $/ \mathrm{mL})$, and streptomycin $(100 \mu \mathrm{g} / \mathrm{mL})$ at $37{ }^{\circ} \mathrm{C}$ in a $5 \% \mathrm{CO}_{2}$ humidified incubator. Cells were seeded at $1 \times 10^{5}$ cells per well in a 12 -well plate the day before transfection. Cells were co-transfected with a total of $500 \mathrm{ng}$ of the psiCHECK-2 luciferase reporter construct or empty psiCHECK-2 vector, and $40 \mathrm{pmol}$ of negative control of bta-miR-139 mimic or bta-miR-139 mimic (RiboBio Company, Guangzhou, China). Transfections were performed using Lipofectamine 2000. At $24 \mathrm{~h}$ post-transfection, the medium was changed. The cells were grown for an additional $24 \mathrm{~h}$ before the assay was performed.

Firefly and Renilla luminescent signals arising from psiCHECK-2 transfected cells were quantified according to the manufacturer's instructions for the Dual-Luciferase Reporter Assay System (Promega, Madison, WI, USA) with a VICTOR Multilabel Counter luminometer (PerkinElmer, Waltham, MA). Independent experiments were performed 3-5 times.

\section{BMECs culture and transfection}

BMECs were prepared and identified as previously described [23]. In brief, the tissue samples from lactating 
Holstein cows were minced with surgical scissors and transferred to cell culture bottles coated with collagen. Mammary tissue pieces were cultured in Dulbecco's modified Eagle's medium F-12 (DMEM/F12) (Gibco, Rockville, Maryland, USA) and supplemented with $10 \%$ FBS, penicillin (100 units $/ \mathrm{mL})$, and streptomycin $(100 \mu \mathrm{g} / \mathrm{mL})$ at $37{ }^{\circ} \mathrm{C}$ in a $5 \% \mathrm{CO}_{2}$ humidified incubator. BMECs and fibroblasts were separated by selective trypsinization (0.25\% trypsin). Pure BMECs were obtained after four passages and identified by immunofluorescence for Cytokeratin-18 and $\beta$-casein.

The BMECs were seeded at $3 \times 10^{5}$ cells per well in a 6-well plate and grown for $24 \mathrm{~h}$. Then, the cells were transfected with 100 pmol of negative control of btamiR-139 mimic (Mimic NC), bta-miR-139 mimic, negative control of bta-miR-139 inhibitor (Inhibitor NC) or bta-miR-139 inhibitor (RiboBio Company, Guangzhou, China) using Lipofectamine 2000 (Invitrogen Life Technologies, Carlsbad, CA, USA) according to the manufacturer's instructions. The control group was only transfected with Lipofectamine 2000. Opti-MEM (Reduced Serum Medium) (Gibco, Rockville, Maryland, USA) was used as medium. Approximately $5 \mathrm{~h}$ after transfection, the medium was changed to DMEM/F12 supplemented with $10 \%$ FBS.

\section{RNA extraction and quantitative real-time polymerase chain reaction assay}

Total RNA was extracted from frozen tissues or BMECs using Trizol reagent (Invitrogen, Carlsbad, CA, USA) according to the manufacturer's instructions. RNA extraction from BMECs was performed $24 \mathrm{~h}$ post-transfection. RNA integrity was assessed by electrophoresis on $1 \%(w / v)$ agarose gels. The ratio of the optical densities measured at 260 and $280 \mathrm{~nm}$ by an ND-1000 spectrophotometer (NanoDrop Technologies, USA) was $>1.8$ for all RNA samples.

To determine the relative amount of miR-139 or U6 transcripts, cDNA was synthesized using M-MLV reverse transcriptase (Invitrogen, Carlsbad, CA, USA) according to the manufacturer's instructions. Primer sets used in reverse transcription and quantitative Real-time Polymerase Chain Reaction(qPCR) for bta-miR-139 or U6 were purchased from GenePharma Company (Shanghai, China). To determine the relative amount of GHR or IGF1R transcripts, cDNA was synthesized using the PrimeScript ${ }^{\mathrm{TM}}$ RT reagent kit (TaKaRa, Ostu, Japan). QPCR reactions were performed using SYBR Premix Ex $\mathrm{Taq}^{\mathrm{TM}}$ (TaKaRa, Ostu, Japan) and the ABI PRISM 7300 Real-Time PCR System (Applied Biosystems, Foster City, CA, USA). The primers were designed by Primer Premier 5.0 software. The primers used were as follows-GHR: forward 5'-CGTGGACAACGCTTACT -3' and reverse 5'-AAGGGTTTCTGTGGTGAT -3'; IGFIR: forward 5'-AGAAGCAGGCGGAGAAGGA-3' and reverse $5^{\prime}$-AAGGGTTTCTGTGGTGAT $-3{ }^{\prime} ; \beta$-actin: forward 5'-TTAGCTGCGTTACACCCTT -3 ' and reverse 5'-GTCACCTTCACCGTTCCA -3'. All reactions were performed in triplicate. The expression of miR-139 was normalized to U6. Gene expression was normalized to $\beta$-actin. The stability of U6 and $\beta$-actin were confirmed under the experimental conditions. The relative levels of miRNA or mRNA were determined using the $2^{-\Delta \Delta C t}$ method.

\section{Western blotting analysis}

Western blotting analysis was performed as previously described [24]. Briefly, BMECs were harvested on ice using RIPA buffer (Beyotime Biotechnology, Shanghai, China) to which protease inhibitors had been added (2 mM PMSF, $5 \mathrm{mM} \mathrm{Na} \mathrm{PO}_{4}, 5 \mathrm{mM} \mathrm{NaF}$, and complete protease inhibitor) at $48 \mathrm{~h}$ post-transfection and then homogenized by ultrasonication. The BCA protein assay was used to measure protein concentration in the samples. Approximately $50 \mu \mathrm{g}$ total protein was subjected to a $10 \%$ SDS-PAGE. The separated proteins were transferred to a nitrocellulose membrane and incubated in blocking buffer. Membranes were then incubated with primary antibodies overnight at $4{ }^{\circ} \mathrm{C}$, followed by incubation with horseradish peroxidase-conjugated secondary antibodys against mouse, rabbit or goat (ZSGB-BIO, Beijing, China) at room temperature for $1 \mathrm{~h}$. Protein bands were visualized using an ECL system. The following primary antibodies were purchased from Santa Cruz Biotechnology: antibodies for phosphorylated signal transducer and activator of transcription-5 (p-STAT5) (1:200 dilution, sc-11,761), signal transducer and activator of transcription-5 (STAT5) (1:200 dilution, SC-836), protein kinase B (also known as AKT1) (1:200 dilution, SC-1618), phosphorylated AKT1 (1:200 dilution, sc135,650), phosphorylated p70-S6 Kinase (1:200 dilution, p-p706SK) (sc-11,759), p70-S6 Kinase (p706SK) (1:200 dilution, sc-230), Cyclin D1 (1:200 dilution, sc-718), and $\beta$-actin (1:400 dilution, sc-47,778); from Cell Signaling Technology: antibodies for IGF1R (1:1000 dilution, \#3027), phosphorylated mammalian target of rapamycin (p-mTOR) (1:1000 dilution, \#2971) and mammalian target of rapamycin (mTOR) (1:1000 dilution, \#2972); and from Abbiotec Company: $\beta$-casein antibody (1:200 dilution, \#251309). $\beta$-actin was used as an endogenous reference gene. All experiments were performed in triplicate. Western blotting data were scanned and quantified using Bandscan5.0 software.

\section{3-(4,5-Dimethylthiazol-2-yl)-2,5- diphenyltetrazolium bromide assay}

BMECs were cultured in 96-well plates $\left(1 \times 10^{4}\right.$ cells per well). At 24, 48, 72, or $96 \mathrm{~h}$ post-transfection with Lipofectamine 2000 (Control), negative control of bta-miR- 
139 mimic (Mimic NC), bta-miR-139 mimic, negative control of bta-miR-139 inhibitor (Inhibitor NC) or btamiR-139 inhibitor, cell proliferation was assayed using (3-(4,5-Dimethylthiazol-2-yl)-2,5- diphenyltetrazolium bromide) (MTT) dye (Amresco, Colorado, USA). MTT (5 $\mathrm{mg} / \mathrm{ml})$ was added to plates and incubated for $4 \mathrm{~h}$. After that, the medium was discarded and cells were lysed in dimethyl sulfoxide (DMSO) (Amresco, Colorado, USA). Absorbance was measured at $490 \mathrm{~nm}$ using the Bio-Rad iMark 680 microplate reader (Bio-Rad, USA).

\section{Co-transfection of GHR siRNA and miR-139 inhibitor}

Small interfering RNA (siRNA) duplexe targeting bovine GHR mRNA and a negative control were designed and synthesized by RiboBio Company (Guangzhou, China). The siRNA sequences were as follows: forward 5' $-\mathrm{CCA}$ GUCCUAGAGACAAAUU dTdT -3' and reverse -3' dTdT GGUCAGGAUCUCUGUUUAA 5'. The targeting sequence in GHR was as follow: CCAGTCCTAGAGA CAAATT. BMECs were seeded in a 6-well plate at a density of $3 \times 10^{5}$ cells per well in DMEM/F12 without antibiotics at $\sim 80-90 \%$ confluence. They were cotransfected with negative control of bta-miR-139 inhibitor or bta-miR-139 inhibitor, and negative control of GHR siRNA or GHR siRNA ( $2 \mu \mathrm{g}$ per $10^{5}$ cells). Lipofectamine 2000 was used according to the manufacturer's protocol. Approximately $6 \mathrm{~h}$ after transfection, the medium was changed to DMEM/F12. RNA extraction from BMECs was performed $24 \mathrm{~h}$ post-transfection, and protein extraction was performed $48 \mathrm{~h}$ post-transfection.

\section{Statistical analysis}

Statistical analyses were performed using SPSS19.0 software. All data are reported as the mean \pm SEM of three independent experiments. Comparisons between treatments were made by $t$ tests for two groups or ANOVA if there were more than two groups. Statistical significance was considered at $P<0.05$.

\section{Results}

Expression of miR-139 in dairy cow mammary tissues

To determine the expression of miR-139 in dairy cow mammary glands, we used qPCR to measure levels of the mature miR-139 in mid-pregnancy and mid-lactation mammary tissues of dairy cows. U6 was used for normalization. The result showed that miR-139 expression in mid-lactation bovine mammary tissues was significantly reduced compared with that in mid-pregnancy tissues $(P<0.05)$ (Fig. 1). This result suggested that down-regulation of miR-139 may be important in lactation of dairy cows.

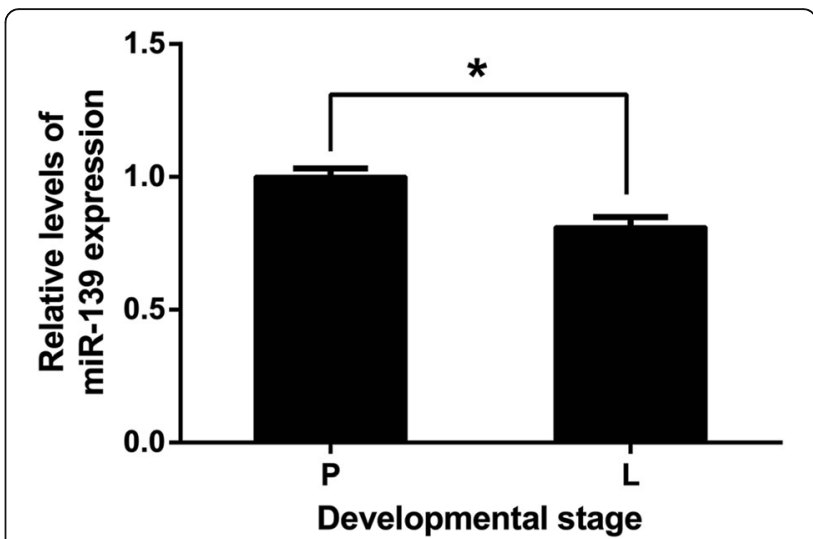

Fig. 1 Expression of miR-139 in Holstein cow mammary gland. Mammary tissues were from mid-pregnancy $(P)(n=3)$ or mid-lactation $(L)(n=3)$ cows. MiR-139 expression was normalized to U6. Results are shown as means \pm SEM from three independent experiments. Statistical analysis was conducted using the $t$ test."*", statistical significance was considered at $P<0.05$

MiR-139 directly targets 3'-UTRs of GHR and IGF1R in dairy cow

Through a target prediction algorithm (TargetScan, http://www.targetscan.org), we found that GHR and $I G F 1 R$ are potential target genes of miR-139 in cow. It is known that miRNAs function by binding to the 3 '-UTR of their target mRNAs and inhibiting their expression. Then, to verify the binding of miR-139 to GHR and $I G F 1 R$, we performed a dual luciferase reporter assay in HeLa cells using the psiCHECK-2 vector and engineered luciferase reporter constructs containing the wild-type (WT) or mutant (Mut) 3'-UTR of the GHR or IGF1R gene, separately (Fig. 2a and b). Compared with cotransfection of negative control of miR-139 mimic with the luciferase reporter gene linked to the wild-type 3 'UTR of GHR or IGF1R, co-transfection of miR-139 mimic with the wild-type construct of GHR or IGF1R 3 '-UTR strongly inhibited the luciferase activity. Luciferase activity was decreased in these cells by $53.5 \%$ or $51.7 \%$, respectively $(P<0.05)$ (Fig. $2 \mathrm{c}$ and $\mathrm{d})$. In addition, luciferase activity was not altered by co-transfection of negative control of $\mathrm{miR}-139 \mathrm{mimic} / \mathrm{miR}-139 \mathrm{mimic}$ with the mutant construct of GHR or IGF1R $3^{\prime}$-UTR in which the miR-139 binding sequence was mutated $(P>$ 0.05 ) (Fig. $2 \mathrm{c}$ and $\mathrm{d}$ ). These results revealed that miR139 directly binds to the 3 '-UTRs of GHR and IGF1R.

\section{MiR-139 decreases mRNA levels of GHR and IGF1R in BMECs}

To investigate whether expression of GHR and IGF1R is regulated by miR-139, we measured mRNA levels of miR-139, GHR and IGF1R by qPCR, following overexpression or silencing of miR-139 in cultured BMECs. BMECs were transfected with negative control of miR- 

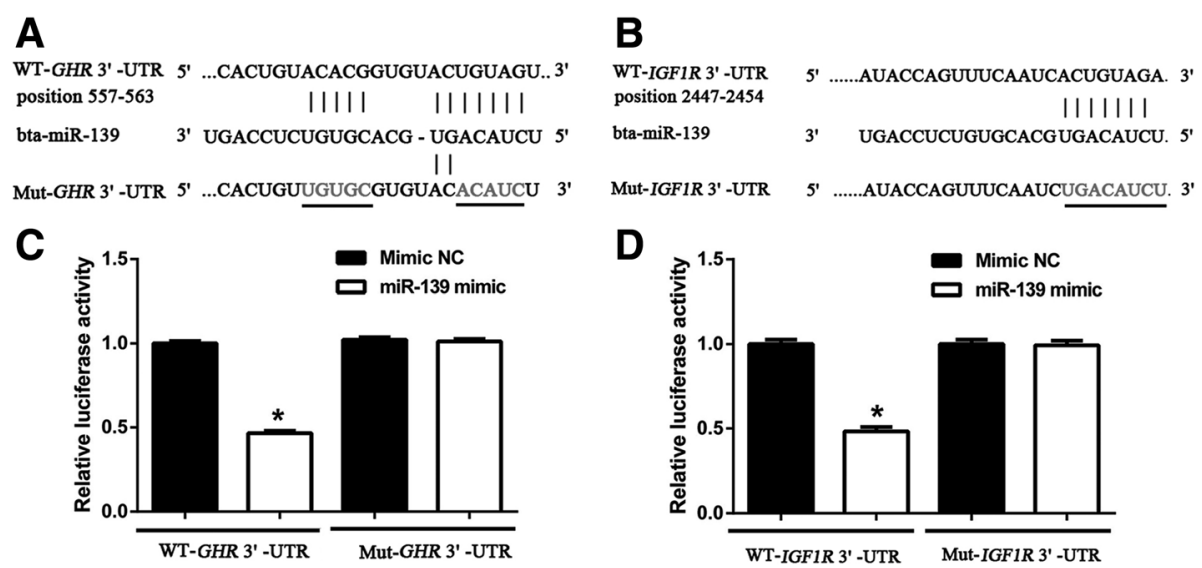

Fig. 2 Identifying target genes of miR-139 via the dual luciferase reporter assay. a Bta-miR-139 binding sites in the 3'-UTR of GHR. Ten nucleotides (underlined) were mutated in luciferase reporter plasmids carrying GHR 3'-UTR. b Bta-miR-139 binding sites in the 3'-UTR of IGFIR. Eight nucleotides (underlined) were mutated in the luciferase reporter plasmids carrying IGFIR 3'-UTR. c Luciferase activity of reporter plasmids carrying wild-type or mutant GHR 3'-UTR in HeLa cells, in response to co-transfection with miR-139. $\mathbf{d}$ Luciferase activity of reporter plasmids carrying wild-type or mutant IGFIR 3'-UTR in HeLa cells, in response to co-transfection with miR-139. Results are shown as means \pm SEM from three independent experiments. Statistical analysis was conducted using one-way ANOVA." ${ }^{\prime *}$, statistical significance was considered at $P<0.05$

139 mimic, miR-139 mimic, negative control of miR-139 inhibitor or miR-139 inhibitor, separately. The Control group was transfected with Lipofectamine 2000. As expected, compared with the Control group, overexpression of miR-139 significantly increased the level of miR-139 $(P<0.05)$ (Fig. 3a), whereas silencing miR-139 decreased it $(P<0.05)$ (Fig. 3b). Then, we examined mRNA levels of GHR or IGF1R with each treatment. Our qPCR results revealed that over-expression of miR139 inhibited GHR expression by approximately $32 \%$ compared with the Control group $(P<0.05)$ (Fig. 3c). However, miR-139 inhibitor treatment increased the level of GHR by approximately 37\% compared with the Control group $(P<0.05)$ (Fig. 3d). Our qPCR results also showed that over-expression of miR-139 significantly decreased the expression of IGF1R by approximately $21 \%$ compared with the Control group $(P<0.05)$ (Fig. 3e), whereas loss of miR-139 increased the expression of IGF1R by approximately $73 \%$ compared with the Control group $(P<0.05)$ (Fig. 3f). Taken together, these results confirmed that miR-139 negatively regulates mRNA levels of GHR and IGF1R.

\section{Effects of miR-139 in BMECs}

The mammary gland in female mammals is responsible for milk production. To determine the role of miR-139 in milk production, we measured the protein levels of $\beta$-casein after over-expression or silencing of miR-139 in cultured BMECs. We found that overexpression of miR-139 decreased the protein level of $\beta$-casein compared with the Control group $(P<0.05)$ (Fig. 4a and c), whereas silencing miR-139 increased it $(P<0.05)$ (Fig. $4 \mathrm{~b}$ and $\mathrm{d})$.
Milk production is determined by the number of secretory mammary epithelial cell [25]. Then, we assessed the role of miR-139 on the proliferation of BMECs. The MTT assay revealed that miR-139 significantly decreased the number of BMECs at 48 and $72 \mathrm{~h}$ post-transfection with miR-139 mimic in comparison with the Control and Mimic NC groups $(P<$ 0.05) (Fig. 5a), whereas silencing miR-139 increased the proliferation of BMECs at 48 and $72 \mathrm{~h}$ posttransfection in comparison with the Control and Inhibitor NC groups $(P<0.05)$ (Fig. 5b). These results revealed that miR-139 inhibits cell growth in BMECs.

Next, to explore the underlying molecular mechanism of miR-139, we examined the protein levels of key members of the GHR and IGF1R signaling pathways after over-expression or silencing of miR-139 in cultured BMECs. Western blotting analysis showed that, compared with the Control and Mimic NC groups, over-expression of miR-139 reduced protein levels of $\mathrm{p}$ STAT5 and STAT5, which are key members in the GHR signaling pathway $(P<0.05)$ (Fig. $6 \mathrm{a}$ and $\mathrm{c}$ ); IGF1R, pAKT1, AKT1 and Cyclin D1, which are key members in the IGF1R signaling pathway $(P<0.05)$ (Fig. $6 \mathrm{a}$ and $\mathrm{c}$ ); and $\mathrm{p}$-mTOR, mTOR, p-p70S6K and p70S6K, which are key members in both GHR and IGF1R signaling pathways $(P<0.05)$ (Fig. 6a and c). Moreover, compared with the Control and Ihibitor NC groups, silencng miR-139 increased the levels of these proteins $(P<0.05)$ (Fig. $6 \mathrm{~b}$ and $d)$. These results demonstrated that miR-139 downregulates $\beta$-casein synthesis and mammary epithelial cells proliferation by influencing the GHR and IGFIR signaling pathways in BMECs. 

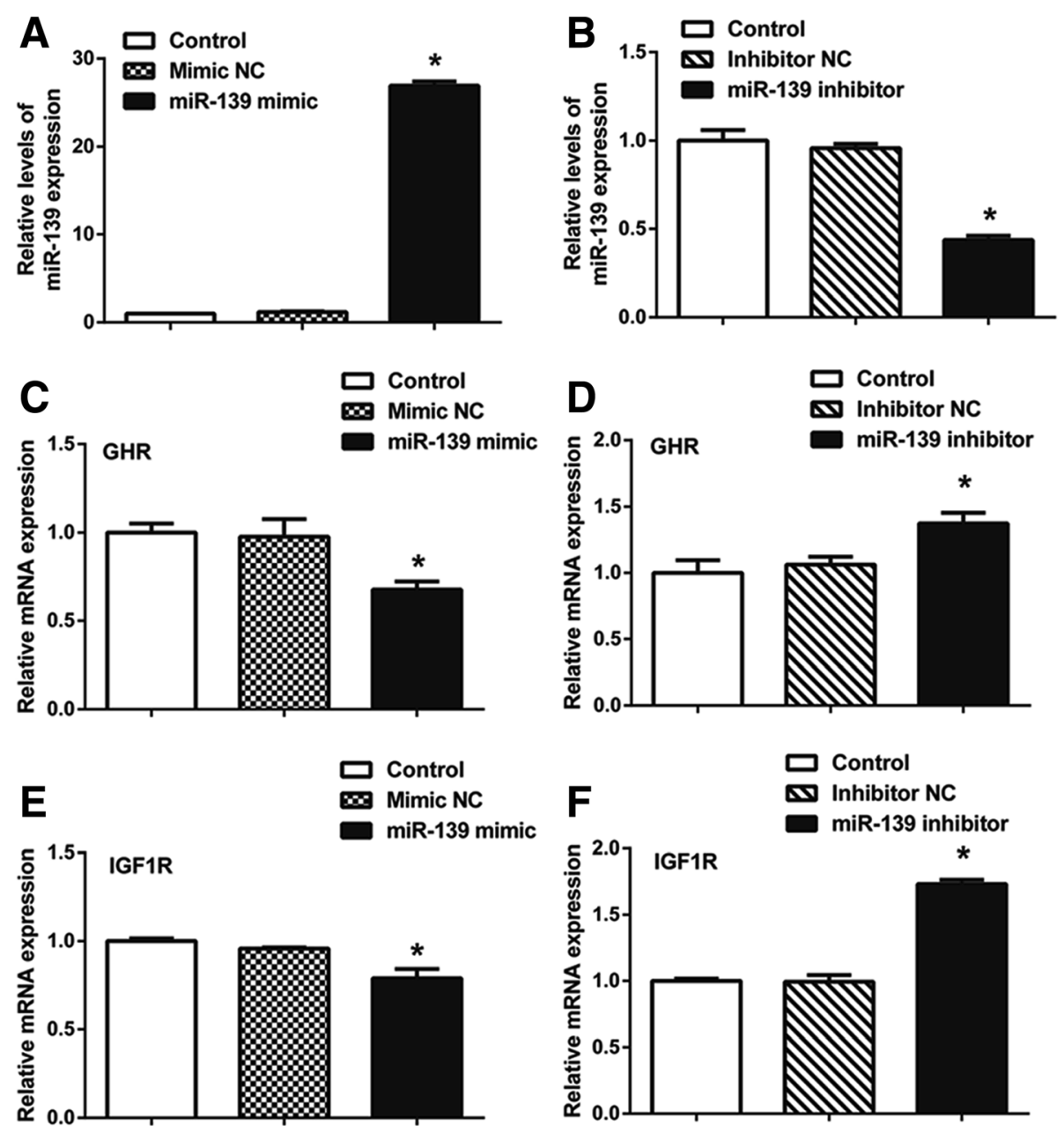

Fig. 3 GHR and IGFIR are target genes of miR-139. BMECs were transfected with negative control of miR-139 mimic (Mimic NC), miR-139 mimic, negative control of miR-139 inhibitor (Inhibitor NC) or miR-139 inhibitor using Lipofectamine 2000. The Control group was only transfected with Lipofectamine 2000. MRNA levels of miR-139 (a, b), GHR (c, d) and IGFIR (e, f) were examined by qPCR at $24 \mathrm{~h}$ post-transfection. MiR-139 expression was normalized to U6. GHR and IGFIR expression was normalized to $\beta$-actin. Results are shown as means \pm SEM from three independent experiments. Statistical analysis was conducted using one-way ANOVA."*", statistical significance was considered at $P<0.05$

MiR-139 also regulates $\beta$-casein synthesis and IGF1R signaling by influencing $G H R$

We next sought to determine the relationship among miR-139, GHR, and IGF1R. As we have shown, silencing miR-139 significantly increased mRNA levels of GHR ( $P$ $<0.05$ ) (Fig. 7a), and protein levels of IGF1R, p-AKT1, $\mathrm{AKT} 1$, and $\beta$-casein in comparison with the Control group $(P<0.05)$ (Fig. $7 \mathrm{~b}$ and $\mathrm{c}$ ). While, silencing $G H R$ by siRNA significantly decreased the mRNA levels of $G H R$ in comparison with the Control group $(P<0.05)$ (Fig. 7a). Moreover, the loss of GHR expression also contributed to decreased protein levels of IGF1R, p-AKT1, AKT1, and $\beta$-casein in comparison with the Control group $(P<$ 0.05 ) (Fig. $7 \mathrm{~b}$ and $\mathrm{c}$ ). In addition, when GHR and miR139 were both silenced, mRNA level of GHR and protein levels of IGF1R, p-AKT1, AKT1, and $\beta$-casein increased relative to those of the group with silenced GHR $(P<0.05)$
(Fig. 7a-c). These results indicated that IGF1R signaling is also regulated by GHR. However, the expression and effects of GHR can be influenced by miR-139.

\section{Discussion}

The mammary gland gains the function of milk production during lactation [26]. Our previous results from microRNA microarrays showed that the expression of some miRNAs, such as miR-139, is down-regulated on transition from pregnancy to lactation in mammary tissues from mouse [11] and dairy cow (data unpublished). Since miRNAs can trigger inhibition of protein translation $[4,27,28]$, down-regulation of certain miRNAs during lactation indicates that they may play important roles related to lactation [29]. We observed that the expression of miR-139 is reduced in dairy cow mammary gland from pregnancy to lactation. Therefore, we 
A

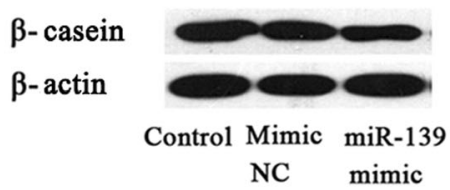

B

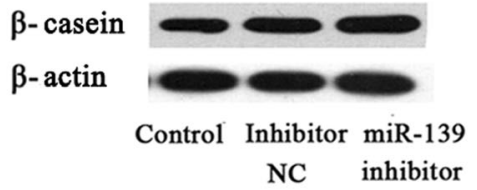

C

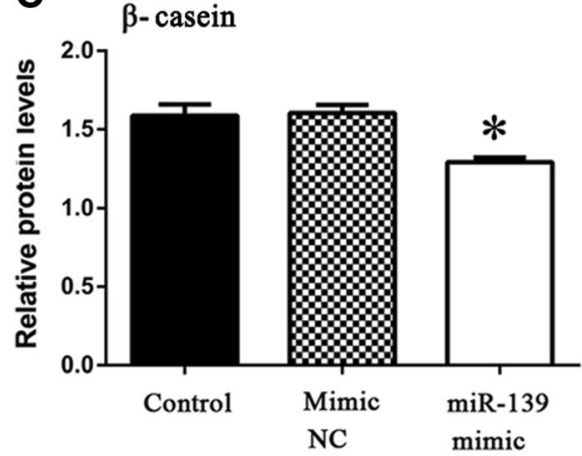

D

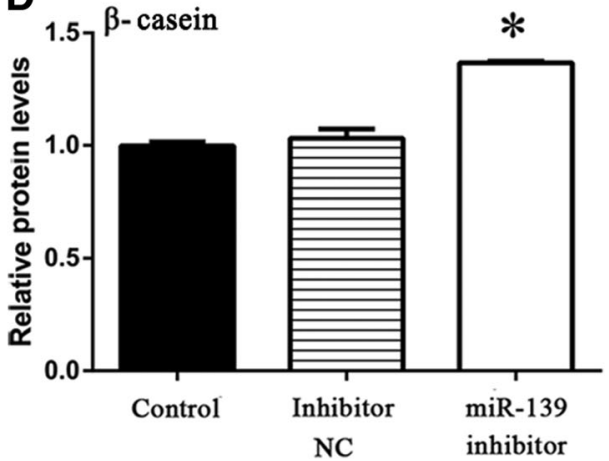

Fig. 4 MiR-139 inhibits protein levels of $\beta$-casein in BMECs. a BMECs were transfected with Lipofectamine 2000 (Control), negative control of miR-139 mimic (Mimic NC) or miR-139 mimic. Western blotting analysis showed the protein levels of $\beta$-casein and $\beta$-actin at $48 \mathrm{~h}$ post-transfection. $\mathbf{b}$ BMECs were transfected with Lipofectamine 2000 (Control), negative control of miR-139 inhibitor (Inhibitor NC) or miR-139 inhibitor. Western blotting analysis showed the protein levels of $\beta$-casein and $\beta$-actin at $48 \mathrm{~h}$ post-transfection. $\mathbf{c}$ Relative protein levels were quantified by analyzing scanned blots in panel a using Bandscan5.0 software. $\beta$-actin was used as a loading control. d Relative protein levels were quantified by analyzing scanned blots in panel $\mathbf{b}$ using Bandscan5.0 software. $\beta$-actin was used as a loading control. For $\mathbf{c}$ and $\mathbf{d}$, results are shown as means $\pm S E M$ from three independent experiments. Statistical analysis was conducted using one-way ANOVA. "**", statistical significance was considered at $P<0.05$

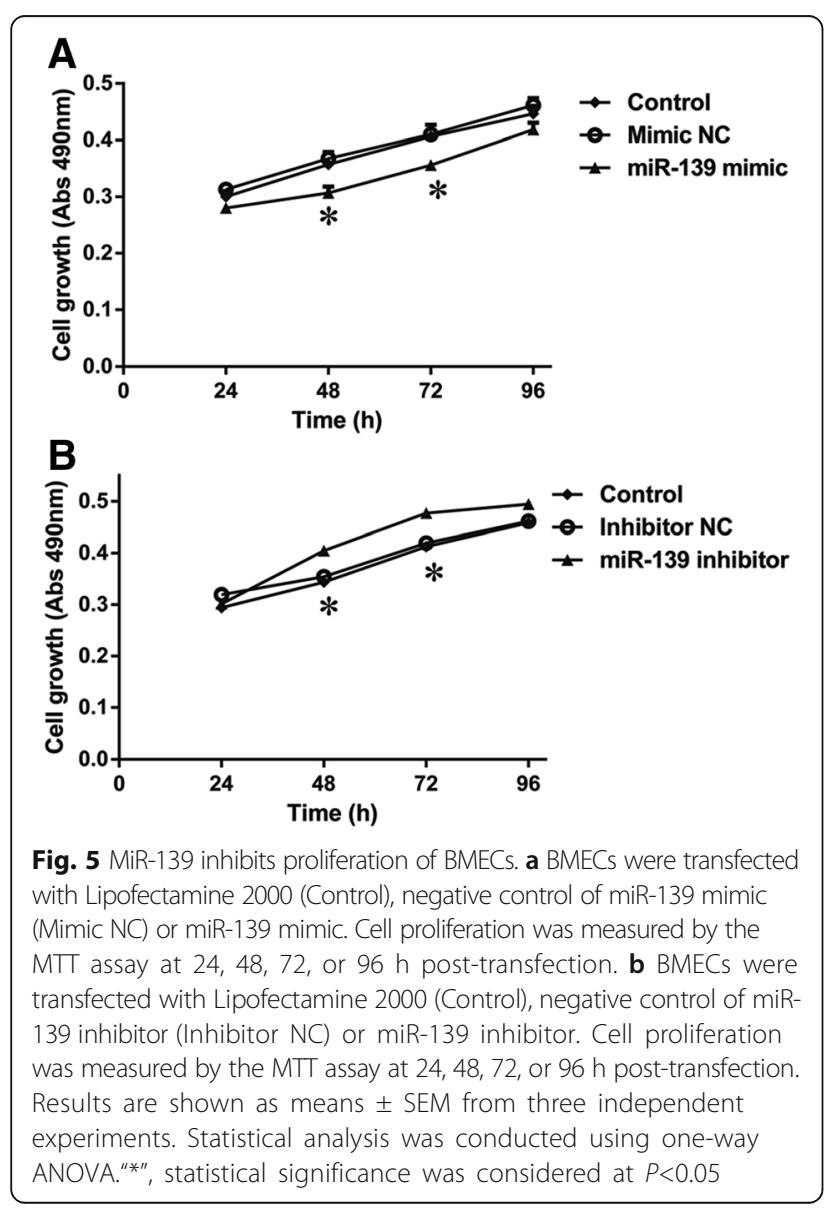

hypothesized that miR-139 may be involved in lactation of dairy cows and tested this hypothesis. Out of potential target genes for miR-139, we prioritized GHR and IGF1R for further studies based on their roles in lactation processes. Our dual luciferase reporter assay indicated that miR-139 binds to GHR and IGF1R by their 3 '-UTRs. Furthermore, we observed that mRNA levels of GHR and IGF1R were inversely correlated with miR-139 expression in cultured BMECs. These results confirmed that GHR and IGF1R are target genes of miR-139 in dairy cow mammary gland.

GHR activates signaling pathways including the Janus kinase2/signal transducer and activator of transcription (JAK2/STAT), mitogen activated protein kinase (MAPK), and the phosphatidylinositol-3-kinase (PI3K) pathways [14]. In particular, the JAK-STAT5 pathway is the main pathway through which GH influences the expression of genes encoding milk proteins such as $\beta$ casein, an important differentiation marker in mammary epithelial cells [16, 30, 31]. Moreover, GH influences protein metabolism through changes in the mTOR signaling pathway in bovine mammary gland [9]. It is worth noting that the mTOR signaling pathway can also be influenced by IGF1R. There are two classical signaling pathways regulated by IGF1R, the PI3K-AKT and RAS/ RAF/MAPK pathways [32]. IGF1R regulates protein synthesis through the PI3K-AKT1-mTOR pathway [33]. After phosphorylation of mTOR, the mTOR pathway regulates the translation process by directly 

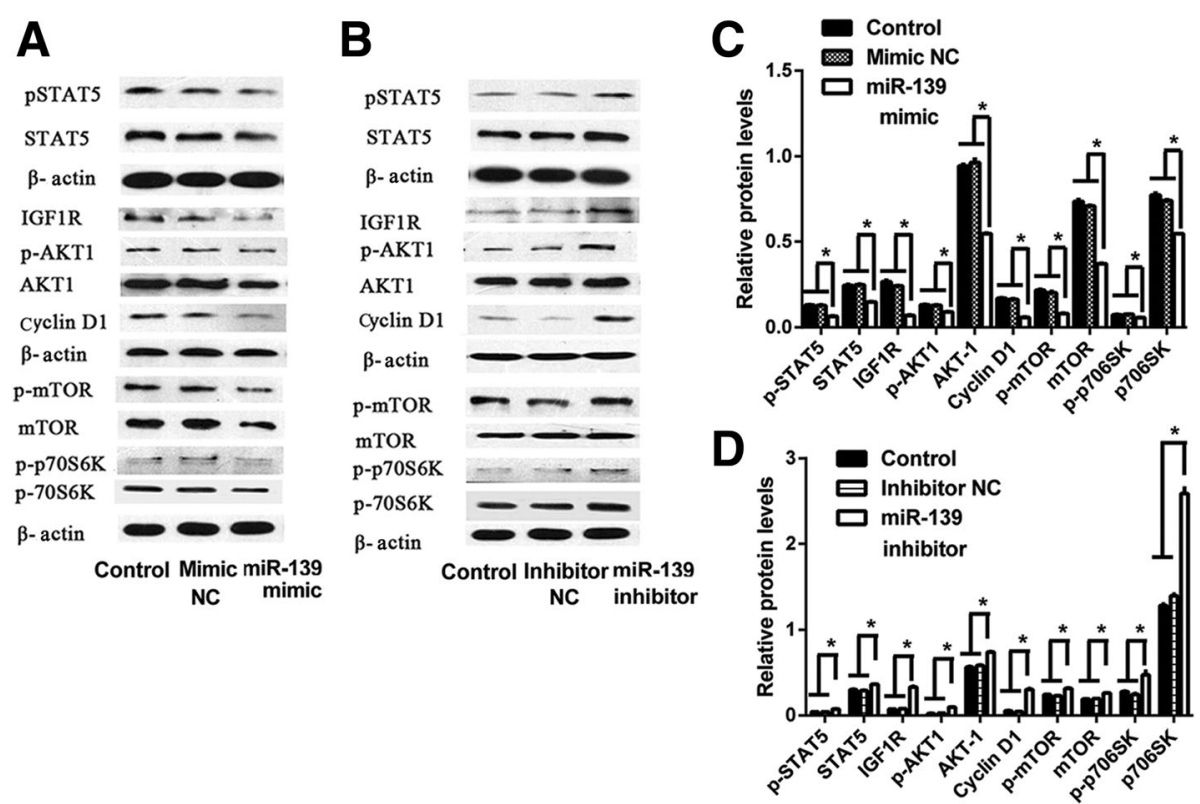

Fig. 6 MiR-139 reduces protein levels of major downstream members of GHR and IGF1R signaling in BMECs. a BMECs were transfected with Lipofectamine 2000 (Control), negative control of miR-139 mimic (Mimic NC) or miR-139 mimic. Western blotting analysis showed the protein levels of p-STAT5, STAT5, IGF1R, p-AKT1, AKT1, Cyclin D1, p-mTOR, mTOR, p-p70S6K, p70S6K and $\beta$-actin at $48 \mathrm{~h}$ post-transfection. b BMECs were transfected with Lipofectamine 2000 (Control), negative control of miR-139 inhibitor (Inhibitor NC) or miR-139 inhibitor. Western blotting analysis showed the protein levels of p-STAT5, STAT5, IGF1R, p-AKT1, AKT1, Cyclin D1, p-mTOR, mTOR, p-p70S6K, p70S6K and $\beta$-actin at $48 \mathrm{~h}$ post-transfection. c Relative protein levels were quantified by analyzing scanned blots in panel a using Bandscan 5.0 software. $\beta$-actin was used as a loading control. $\mathbf{d}$ Relative protein levels were quantified by analyzing scanned blots in panel b using Bandscan5.0 software. $\beta$-actin was used as a loading control. For $\mathbf{c}$ and $\mathbf{d}$, results are shown as means \pm SEM from three independent experiments. Statistical analysis was conducted using one-way ANOVA. "*", statistical significance was considered at $P<0.05$

phosphorylating the translational regulators eukaryotic translationinitiation factor $4 \mathrm{E}$ (eIF4E) binding protein 1 (4E-BP1) and S6 kinase 1 (S6 K1), which in turn promote protein synthesis $[19,34]$. In our study, we revealed that miR-139 reduced protein levels of $\beta$-casein. In addition, we found that miR-139 inhibited not only the activity of downstream signaling molecules of the GHR and IGF1R pathways, but also the total protein levels of key molecules of these pathways. This phenomenon has also been found by others [24, 35]. These observations suggest that miR-139 likely inhibits synthesis of $\beta$-casein through GHR and IGF1R signaling in BMECs.

In general, the number of secretory mammary epithelial cells determines milk production in a mammary gland, and the balance between proliferation and apoptosis controls the number of secretory mammary epithelial cells. Both proliferation and apoptosis produce effects gradually throughout the lactation period [25]. Previous studies showed that IGF1R can stimulate cell proliferation and inhibit apoptosis through the PI3K/ AKT and MAPK pathways [36]. Our study revealed that miR-139 down-regulated expression of IGF1R and PI3K/ AKT members. Moreover, in our cell proliferation assay, miR-139 significantly inhibited the growth of BMECs.
Cyclin D is a known cell cycle regulator which indirectly stimulates the expression of various cell cycle promoters [37]. Previous studies showed that IGF1R induces elevated protein levels of Cyclin D1 through binding of the lymphoid Enhancer-Binding Factor 1 (LEF1) transcription factor in the nucleus [38]. Then, we determined the expression of Cyclin D1 by western blotting analysis. Our results showed that miR-139 decreased the protein level of Cyclin D1 in cultured BMECs. These results suggest that miR-139 also reduces the number of BMECs via IGF1R signaling.

In this study, we determined that GHR and IGF1R are target genes of miR-139 and that miR-139 inhibits synthesis of $\beta$-casein through down-regulating both GHR and IGF1R signaling. However, there is another connection between GHR and IGF1R. As we know, the binding of GHR to its ligand regulates IGF1 production by activating the STAT5b signaling cascade $[39,40]$. Moreover, postnatal IGF1 production is predominantly regulated by GH [41]. These data indicate that the effect of GHR on the mammary gland may be exerted via IGF1R. Then, we investigated the relationship among miR-139, GHR, and IGF1R. As a result, we revealed that GHR is a regulator of IGF1R signaling because silencing GHR decreased protein levels of $\beta$-casein, IGF1R, and key 


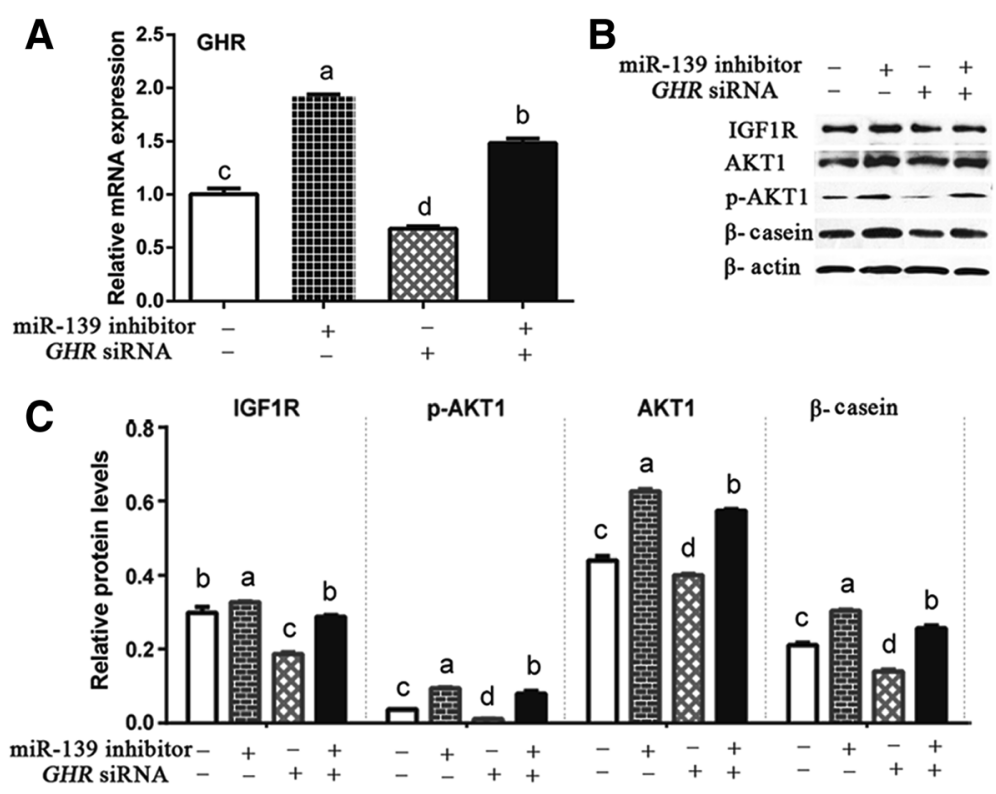

Fig. 7 MiR-139 also regulates synthesis of $\beta$-casein and IGF1R signaling by influencing GHR. BMECs were transfected with (without) GHR siRNA and (or) miR-139 inhibitor. a GHR mRNA levels were examined by qPCR at $24 \mathrm{~h}$ post-transfection in cultured BMECs. GHR expression was normalized to $\beta$-actin. b Western blotting analysis showed the levels of IGF1R, p-AKT1, AKT1, $\beta$-casein, and $\beta$-actin at $48 \mathrm{~h}$ post-transfection in cultured BMECs. c Relative protein levels were quantified by analyzing scanned blots in panel $\mathbf{b}$ using Bandscan 5.0 software. $\beta$-actin was used as a loading control. For $\mathbf{a}$ and $\mathbf{c}$, results are shown as means \pm SEM from three independent experiments. Statistical analysis was conducted using one-way ANOVA. Bars with different lowercase letters are significantly different $(P<0.05)$

members of the IGF1R pathway. Additionally, we found that co-silencing miR-139 and GHR rescued the expression of GHR and GHR silencing effects. These data suggest that miR-139 also regulates synthesis of $\beta$-casein and IGF1R signaling by influencing GHR.

\section{Conclusions}

In conclusion, we have revealed that miR-139 is downregulated in lactating dairy cow mammary glands and that miR-139 inhibits $\beta$-casein synthesis and proliferation by targeting the GHR and IGF1R signaling pathways in BMECs. This finding suggests that miR-139 may act as a potent inhibitor in lactation.

\section{Abbreviations}

AKT1: Protein kinase B; BMEC: Bovine mammary epithelial cell; DIM: Day in milk; DMEM: Dulbecco's modified Eagle's medium; DMSO: Dimethyl sulfoxide; elF4E: Eukaryotic translationinitiation factor 4E; FBS: Fetal bovine serum; GH: Growth hormone; GHR: Growth hormone receptor; IGF1: Type I insulin-like growth factor; IGF1R: Type I insulin-like growth factor receptor; JAK: Janus kinase; LEF1: Lymphoid Enhancer-Binding Factor 1:

MAPK: Mitogen activated protein kinase; mTOR: Mammalian target of rapamycin.; MTT: 3-(4,5-Dimethylthiazol-2-yl)-2,5- diphenyltetrazolium bromide.; Mut: Mutant:; p70S6K: p70-S6 Kinase.; PI3K: Phosphatidylinositol-3kinase.; qPCR: Quantitative Real-time Polymerase Chain Reaction.; SDSPAGE: Sodium dodecyl sulfate polyacrylamide gel electrophoresis.; SEM: Standard error of mean.; STAT5: Signal transducer and activator of transcription-5.; UTR: Untranslated region.; WT: Wild-type.

\section{Acknowledgements}

Not applicable.

\section{Funding}

This work was supported by the National Natural Science Foundation of China (31401093), the Scientific Research Project of Heilongjiang Province Department of Education (12541018), the "Academic Backbone" Project of Northeast Agricultural University (16XG17), and the Open Research Fund for Key Laboratory of Dairy Science (Northeast Agricultural University), Ministry of Education, Heilongjiang Province,China (2015KLDSDF-02).

Availability of data and materials

The authors prefer to show the raw data. The datasets during and/or analysed during the current study are available from the corresponding author on reasonable request.

\section{Authors' contributions}

YC designed the experiments and wrote the manuscript. XS conducted most of the experiments, and analyzed the results. $L J$ performed dual luciferase reporter assay. GY and JA helped XS analyzed the results. QL and XG made the figures and revised the manuscript. CW conceived the idea for the project, and finalized the manuscript. All authors read and approved the final manuscript.

\section{Ethics approval and consent to participate}

All animal care and procedures were performed in accordance with institutional and national guidelines and approved by the Institutional Animal Care and Use Committee of Northeast Agricultural University (China).

Consent for publication

Not applicable.

\section{Competing interests}

The authors declare that they have no competing interests.

\section{Publisher's Note}

Springer Nature remains neutral with regard to jurisdictional claims in published maps and institutional affiliations. 


\section{Author details}

'Key Laboratory of Dairy Science of Education Ministry, Northeast Agricultural University, Harbin, Heilongjiang 150030, People's Republic of China. ${ }^{2}$ College of Life Science, Northeast Agricultural University, Harbin, Heilongjiang 150030, People's Republic of China.

Received: 12 July 2016 Accepted: 9 November 2017

Published online: 25 November 2017

\section{References}

1. Yonemori M, Seki N, Yoshino H, Matsushita R, Miyamoto K, Nakagawa M, Enokida H. Dual tumor-suppressors miR-139-5p and miR-139-3p targeting matrix metalloprotease 11 in bladder cancer. Cancer Sci. 2016;107:1233-42.

2. Qiu G, Lin Y, Zhang H, Wu D. miR-139-5p inhibits epitheliale mesenchymal transition, migration and invasion of hepatocellular carcinoma cells by targeting ZEB1 and ZEB2. Biochem Biophys Res Commun. 2015;463:315-21.

3. Chen X, Gao C, Li H, Huang L, Sun Q, Dong Y, Tian C, Gao S, Dong H, Guan D, Hu X, Zhao S, Li L, Zhu L, Yan Q, Zhang J, Zen K, Zhang CY. Identification and characterization of microRNAs in raw milk during different periods of lactation, commercial fluid, and powdered milk products. Cell Res. 2010;20:1128-37

4. Hasseine LK, Hinault C, Lebrun P, Gautier N, Paul-Bellon R, Van Obberghen E. miR-139 impacts FoxO1 action by decreasing FoxO1 protein in mouse hepatocytes. Biochem Biophys Res Commun. 2009;390:1278-82.

5. Zhang HD, Sun DW, Mao L, Zhang J, Jiang LH, Li J, WuY JH, Chen W, Wang J, Ma R, Cao HX, Wu JZ, Tang JH. MiR-139-5p inhibits the biological function of breast cancer cells by targeting Notch1 and mediates chemosensitivity to docetaxel. Biochem Biophys Res Commun. 2015;465:702-13.

6. Liu R, Yang M, Meng Y, Liao J, Sheng J, Pu Y, Yin L, Kim SJ. Tumorsuppressive function of miR-139-5p in esophageal squamous cell carcinoma. PLoS One. 2013; https://doi.org/10.1371/journal.pone.0077068.

7. Zhang L, Dong Y, Zhu N, Tsoi H, Zhao Z, Wu CW, Wang K, Zheng S, Ng SS, Chan FK, Sung JJ, Yu J. microRNA-139-5p exerts tumor suppressor function by targeting NOTCH1 in colorectal cancer. Mol Cancer. 2014; doi:10.1186/ 1476-4598-13-124.

8. Xu W, Hang M, Yuan CY, FL W, Chen SB, Xue K. MicroRNA-139-5p inhibits cell proliferation and invasion by targeting insulin-like growth factor 1 receptor in human non-small cell lung cancer. Int J Clin Exp Pathol. 2015;8: 3864-70.

9. Wong CC, Wong CM, Tung EK, SL A, Lee JM, Poon RT, Man K, Ng IO. The microRNA miR-139 suppresses metastasis and progression of hepatocellular carcinoma by down-regulating rho-kinase 2. Gastroenterology. 2011;140: 322-31.

10. Krishnan K, Steptoe AL, Martin HC, Pattabiraman DR, Nones K, Waddell N, Mariasegaram M, Simpson PT, Lakhani SR, Vlassov A, Grimmond SM, Cloonan N. miR-139-5p is a regulator of metastatic pathways in breast cancer. RNA. 2013;19:1767-80.

11. Wang C, Li Q. Identification of differentially expressed microRNAs during the evelopment of Chinese murine mammary gland. J Genet Genomics. 2007; 34:966-73.

12. Hayashi AA, Nones K, Roy NC, McNabb WC, Mackenzie DS, Pacheco D, McCoard S. Initiation and elongation steps of mRNA translation are involved in the increase in milk protein yield caused by growth hormone administration during lactation. J Dairy Sci. 2009;92:1889-99.

13. Lombardi S, Honeth G, Ginestier C, Shinomiya I, Marlow R, Buchupalli B, Gazinska P, Brown J, Catchpole S, Liu S, Barkan A, Wicha M, Purushotham A, Burchell J, Pinder S, Dontu G. Growth hormone is secreted by normal breast epithelium upon progesterone stimulationand increases proliferation of stem/progenitor cells. Stem Cell Reports. 2014:2:780-93.

14. Perry JK, Mohankumar KM, Emerald BS, Mertani HC, Lobie PE. The contribution of growth hormone to mammary neoplasia. J Mammary Gland Biol Neoplasia. 2008;13:131-45.

15. Sakamoto K, Komatsu T, Kobayashi T, Rose MT, Aso H, Hagino A, Obara Y. Growth hormone acts on the synthesis and secretion of alpha-casein in bovine mammary epithelial cells. J Dairy Res. 2005;72:264-70.

16. Postel-Vinay MC, Kelly PA. Growth hormone receptor signalling. Bailliere Clin Endocrinol Metab. 1996;10:323-36.

17. Ilkbahar YN, Thordarson G, Camarillo IG, Talamantes F. Differential expression of the growth hormone receptor and growth hormone-binding protein in epithelia and stroma of the mouse mammary gland at various physiological stages. J Endocrinol. 1999;161:77-87.
18. Pal S, Woodford K, Kukuljan S, Ho S. Milk intolerance, Beta-casein and lactose. Nutrients. 2015;7:7285-97.

19. Sciascia Q, Pacheco D, McCoard SA. Increased milk protein synthesis in response to exogenous growth hormone is associated with changes in mechanistic (mammalian) target of rapamycin (mTOR)C1-dependent and independent cell signaling. J Dairy Sci. 2013;96:2327-38.

20. Divisova J, Kuiatse I, Lazard Z, Weiss H, Vreeland F, Hadsell DL, Schiff R, Osborne CK, Lee AV. The growth hormone receptor antagonist pegvisomant blocks both mammary gland development and MCF-7 breast cancer xenograft growth. Breast. Cancer Res Treat. 2006;98:315-27.

21. Felice DL, El-Shennawy L, Zhao S, Lantvit DL, Shen Q, Unterman TG, Swanson SM, Frasor J. Growth hormone potentiates $17 \beta$-estradioldependent breast cancer cell proliferation independently of IGF-I receptor signaling. Endocrinology. 2013;154:3219-27.

22. Fürstenberger $G$, Morant $R$, Senn $H J$. Insulin-like growth factors and breast cancer. Onkologie. 2003;26:290-4.

23. Cui Y, Liu Z, Sun X, Hou X, Qu B, Zhao F, Gao X, Sun Z, Li Q. Thyroid hormone responsive protein spot 14 enhances lipogenesis in bovine mammary epithelial cells. In Vitro Cell Dev Biol Anim. 2015;51:586-94.

24. Zhao F, Liu C, Hao YM, Qu B, Cui YJ, Zhang N, Gao XJ, Li QZ. Up-regulation of integrin a6 $\beta 4$ expression by mitogens involved in dairy cow mammary development. In Vitro Cell Dev Biol Anim. 2015;51:287-99.

25. Murney R, Stelwagen K, Wheeler TT, Margerison JK, Singh K. The effects of milking frequency in early lactation on milk yield, mammary cell turnover, and secretory activity in grazing dairy cows. J Dairy Sci. 2015;98:305-11.

26. Knight $\mathrm{CH}$, Peaker M. Development of the mammary gland. J ReprodFertil. 1982;65:521-36

27. Zhang HD, Jiang LH, Sun DW, Li J, Tang JH. MiR-139-5p:promising biomarker for cancer. Tumour Biol. 2015;36:1355-65.

28. Ruelas DS, Chan JK, Oh E, Heidersbach AJ, Hebbeler AM, Chavez L, Verdin E, Rape M, Greene WC. MicroRNA-155 reinforces HIV latency. J Biol Chem. 2015;290:13736-48.

29. Zhou Y, Gong W, Xiao J, Wu J, Pan L, Li X, Wang X, Wang W, Hu S, Transcriptomic YJ. Analysis reveals key regulators of mammogenesis and the pregnancy-lactationcycle. Sci China Life Sci. 2014;57:340-55.

30. Chiba T, Maeda T, Sanbe A, Kudo K. Serotonin suppresses $\beta$-casein expression via PTP1B activation in human mammary epithelial cells. Biochem Biophys Res Commun. 2016;473:323-8.

31. Postel-Vinay MC, Finidori J. Growth hormone receptor: structure and signal transduction. Eur J Endocrinol. 1995;133:654-9.

32. Chitnis MM, Yuen JS, Protheroe AS, Pollak M, Macaulay VM. The type 1 insulinlike growth factor receptor pathway. Clin Cancer Res. 2008;14:6364-70.

33. lams WT, Lovly CM. Molecular pathways: clinical applications and future direction of insulin-like growth Factor-1 receptor pathway blockade. Clin Cancer Res. 2015;21:4270-7.

34. Laplante M, Sabatini DM. mTOR signaling in growth control and disease. Cell. 2012;149:274-93.

35. Ao J, Wei C, Si Y, Luo C, Lv W, Lin Y, Cui Y, Gao X. Tudor-SN Regulates milk synthesis and proliferation of bovine mammary epithelial cells. Int J Mol Sci. 2015;16:29936-47.

36. de Groot S, Charehbili A, van Laarhoven HW, Mooyaart AL, Dekker-Ensink NG, van de Ven S, Janssen LG, Swen JJ, Smit VT, Heijns JB, Kessels LW, van der Straaten T, Böhringer S, Gelderblom H, van der Hoeven JJ, Guchelaar HJ, Pijl H, Kroep JR, Dutch Breast Cancer Research Group. Insulin-like growth factor 1 receptor expression and IGF1R 3129G > T polymorphism are associated with response to neoadjuvant chemotherapy in breast cancer patients: results from the NEOZOTAC trial (BOOG 2010-01). Breast Cancer Res. 2016:18:3.

37. Park JH, Darvin P, Lim EJ, Joung YH, Hong DY, Park EU, Park SH, Choi SK, Moon ES, Cho BW, Park KD, Lee HK, Kim MJ, Park DS, Chung IM, Yang YM. Hwanggeumchal sorghum induces cell cycle arrest, and suppresses tumor growth and metastasis through Jak2/STAT pathways in breast cancer xenografts. PLoS One. 2012; doi:10.1371/journal.pone.0040531.

38. Warsito D, Sjöström S, Andersson S, Larsson O, Sehat B. Nuclear IGF1R is a transcriptional co-activator of LEF1/TCF. EMBO Rep. 2012;13:244-50,

39. Hwa V, Nadeau K, Wit JM, Rosenfeld RG. STAT5b deficiency: lessons from STAT5b gene mutations. Best Pract Res Clin Endocrinol Metab. 2011;25:61-75.

40. Feigerlova $E$, Hwa V, Derr MA, Rosenfeld RG. Current issues on molecular diagnosis of GH signaling defects. Endocr Dev. 2013;24:118-27.

41. Hwa V, Fang P, Derr MA, Fiegerlova E, Rosenfeld RG. IGF-I in human growth: lessons from defects in the GH-IGF-I axis. Nestle. Nutrlnst workshop. Ser. 2013;71:43-55. 